\title{
Les instituteurs et l'introduction du travail manuel dans les écoles primaires de garçons du XIXe siècle
}

In: Revue française de sociologie. 1988, 29-2. pp. 275-292.

Citer ce document / Cite this document :

Rougier-Pintiaux Pascale. Les instituteurs et l'introduction du travail manuel dans les écoles primaires de garçons du XIXe siècle. In: Revue française de sociologie. 1988, 29-2. pp. 275-292.

http://www.persee.fr/web/revues/home/prescript/article/rfsoc_0035-2969_1988_num_29_2_2501 


\section{Abstract}

Pascale Rougier-Pintiaux : Schoolmasters and the implementation of manual work in boys primary schools during the 19th century.

The analysis of transactions of the International schoolmasters congress held in Le Havre in 1885, allows one to understand how schoolmasters saw the relationships between the theory and praxis of manual work at a time when vocational education was set up for children of the working-class, and stresses the up-to-dateness of questions raised by the organization of vocational knowledge in today's education. This paper shows the permanence of attitudes towards transmission of vocational knowledge through the school, and the limits of school efficiency in the achievement of setting a vocational education system rooted in the original culture of working-class children.

\section{Zusammenfassung}

Pascale Rougier-Pintiaux : Die Volksschullehrer und die Einführung der Werksarbeit in den Knabenvolksschulen des 19. Jhdt.

Die Analyse der Berichte, die beim Internationalen Volksschullehrerkongress in Le Havre 1885 vorgelegt wurden, hat. zu einem Zeitpunkt an dem in Frankreich die Berufsausbildung für die Kinder der Arbeiterklassen sich organisierte, zum Verständnis beigetragen, wie die Volksschullehrer die Beziehungen zwischen Theorie und Praxis der Werksarbeit seinerzeit verstanden, und zeigl ausserdem die Aktualität der Fragen, die durch die Organisation des Berufswissens im heutigen Unterricht aufgeworfen werden. Diese Untersuchung zeigt das Fortleben verschiedener Einstellungen zur schulischen Ubermittlung der Berufskenntnisse und damit die Wirksamkeitsgrenzen der Schule zur erfolgsreichen Berufslehre, die als Verlängerung der Herkunftskultur der Kinder der Arbeiterklasse betrachtet würde.

\section{Resumen}

Pascale Rougier-Pintiaux : El magisterio y la introducción del trabajo manual en las escuelas primarias de niños en el siglo XIX.

El análisis de los informes presentados en el Congreso internacional de maestros de escuela primaria en Le Havre (1885), permitió comprender en el momento que se organize) en Francia la enseñanza profesional para los niños de las clases populares, cómo los maestros plantearon las relaciones entre la teoria y la práctica del trabajo manual y revela la actualidad de los problemas que plantea la organizacion del saber profesional en la enseñanza actualmente. Este estudio muestra la permanencia de ciertas actitudes con respecto a la transmición escolar de los conocimientos profesionales y de allí, los limites de la eficacidad de la institución escolar para lograr una enseñanza profesional que sea concebida en el prolongamiento de la cultura de origen de los niños de las clases populares.

\section{Résumé}

L'analyse de mémoires présentés au Congrès international des instituteurs du Havre en 1885 a permis de comprendre, au moment où s'organise en France l'enseignement professionnel à l'intention des enfants des classes populaires, comment les instituteurs posent les rapports entre la théorie et la pratique du métier manuel et fait apparaître l'actualité des questions que soulève l'organisation des savoirs professionnels dans l'enseignement d'aujourd'hui. Cette étude montre la permanence de certaines attitudes à l'égard de la transmission scolaire des connaissances professionnelles et, par là, les limites de l'efficacité de l'institution scolaire à réussir un enseignement professionnel qui soit conçu dans le prolongement de la culture d'origine des enfants des classes populaires.

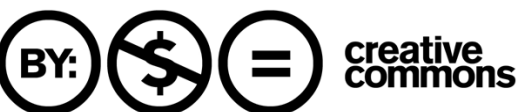




\title{
Les instituteurs et l'introduction du travail manuel dans les écoles primaires de garçons du XIX ${ }^{e}$ siècle*
}

\begin{abstract}
RÉSLMÉ
L'analyse de mémoires présentés au Congrès international des instituteurs du Havre en 1885 a permis de comprendre, au moment où sorganise en France lenseignement professionnel à lintention des enfants des classes populaires, comment les instituteurs posent les rapports entre la théorie et la pratique du métier manuel et fait apparaître l'actualité des questions que soulève lorganisation des savoirs professionnels dans l'enseignement d’aujourd'hui. Cette étude montre la permanence de certaines attitudes à l’égard de la transmission scolaire des connaissances professionnelles et. par là, les limites de lefficacité de linstitution scolaire à réussir un enseignement professionnel qui soit conçu dans le prolongement de la culture dorigine des enfants des classes populaires.
\end{abstract}

Le travail manuel a été introduit à l'école primaire à une époque où s'organise en France l'enseignement professionnel à l'intention des classes populaires.

Nous nous sommes d'abord demandé quelle signification prenait dans ce contexte l'institution de ce nouvel enseignement. Puis, essentiellement à partir de la lecture de mémoires déposés en 1885 lors du Congrès international des instituteurs du Havre (1), nous avons analysé dans quel esprit cet enseignement a été conçu et quelle forme il lui a été donné. Enfin, compte tenu de l'orientation définitive que les instituteurs donneront à cet enseignement, il s'agira de comprendre pourquoi l'institution

* Je remercie J. Testanière, dont laide continue m’a été précieuse dans la préparation de cel article. ainsi que F. Héran pour ses remarques et conseils lors de la mise au point définitive de ce texte.

Une partie de ce travail a fait lobjet dune communication au colloque "Classes populaires et pédagogie ". Rouen. 14-16 mars 1985.

(1) La base matérielle de notre travail est constituée par la lecture de 48 mémoires d'instituteurs. conservés aux archives municipales du Havre, rédigés sur ce sujet à loccasion du premier Congrès international des instituteurs qui se tint au Havre du 6 au 9 septembre 1885. à l'initiative de J. Siegfried. maire du Havre, et en accord avec ladministration primaire -- R. Goblet. ministre de l'Instruction publique. présidera la première séance de ce Congrès qui rassemblera 2400 instituteurs. 
scolaire, à son niveau primaire, n'a pas su ou pu saisir la chance que lui offrait le travail manuel de se réconcilier avec les enfants des classes populaires en leur donnant un enseignement qui soit le prolongement de la culture du travail acquise dans leurs familles (2).

\section{I. - Le travail manuel à l'école primaire : un enseignement créé à l'intention des classes populaires}

Le travail manuel a été introduit à l'école primaire par la loi du 28 mars 1882 qui institue "les travaux manuels et l'usage des principaux métiers". Cet enseignement n'avait pas pour but de constituer un premier degré d'enseignement professionnel en développant dès l'école primaire l'aptitude de l'élève à une activité particulière (3) mais, dégagé de toute préoccupation professionnelle, il devait développer chez l'élève l'habileté manuelle à tous les métiers, préparant en quelque sorte l'entrée à l'école manuelle d'apprentissage, que la loi du 20 novembre 1880 venait de mettre au nombre des écoles primaires publiques. L'introduction de cet enseignement à l'école primaire s'inscrit en effet dans un vaste projet d’organisation d'un enseignement professionnel à l'intention des enfants des classes populaires, défendu par les républicains, qui marqua si profondément le développement de l'enseignement technique en France (4) et dont la création à Vierzon, par le décret du 9 juillet 1881 , de la première école nationale d'enseignement primaire supérieur et d'enseignement préparatoire à l'apprentissage devait servir de modèle. Dans le discours prononcé à l'occasion de la pose de la première pierre de cette école, J. Ferry présente l'école professionnelle comme la chance des ouvriers: en leur apportant la culture de leur métier, l'école leur donnera les moyens de se libérer de ses asservissements. Pour atteindre cet objectif, l'atelier scolaire doit être radicalement différent de l'atelier industriel et les programmes doivent respecter le principe qui veut "que dans une démocratie l'enseignement primaire (...) doit consister d'abord en une éducation générale sans laquelle il n'y a pas de spécialité durable, pas d'enseignement professionnel solide et sérieux $\gg(5)$. Cette conception de l'enseignement

(2) Les travaux manuels agricoles sont exclus de cette étude.

(3) "Nous ne demandons pas (...) que lécole primaire devienne une école professionnelle: nous croyons quion nen doit sortir ni serrurier, ni vigneron: (...) mais nous croyons que l'enseignement scientifique ne doit pas rester dans le domaine de la théorie pure. que les applications pratiques aux diverses industries doivent y tenir une grande place. " (Paul Bert, rapporteur du projet de loi du 28 mars 1882, cité par Leblanc, 1895. p. 5)

Les références bibliographiques se trouvent in fine.

(4) Voir à ce sujet les débats entre $F$. Buisson et G. Ollendorf, cités par Prost. 1968. pp. 318-319; et Léon. 1961 et 1965.

(5) Discours de J. Ferry, Revue pédagogique, $\mathrm{I}^{\text {er }}$ semestre 1883, pp. 461-466. 
manuel fait penser à la défense d'un humanisme technique qui s'élabore d'abord en réaction aux abus de l'apprentissage industriel de l'époque (Sandrin, 1982) mais renvoie aussi, dans une période de crise économique intense (Noiriel, 1986), à une volonté d’adapter à long terme la formation des futurs ouvriers aux changements intervenus dans l'entreprise industrielle. A une époque où l'industrie mécanique se développe considérablement, la formation professionnelle, qui convenait bien aux métiers artisanaux du secteur du bâtiment ou de l'ameublement par exemple, n'est plus adaptée aux caractéristiques de l'entreprise nouvelle. La capacité d'adaptation rapide à des tâches parcellaires, la distinction de plus en plus marquée entre le concepteur et l'exécutant, la polyvalence et la mobilité professionnelle exigent de l'ouvrier une qualification générale indépendante des conditions de travail et des matériaux à travailler et s'opposent à la formation traditionnelle qui vise à la possession d'un métier complet (cf. Vincent, 1972, p. 69). De là, semble-t-il, la volonté d'enseigner "les notions générales et communes à tous les métiers " selon une méthode qui, à partir de 1887 , cesse de préconiser la construction d'un objet complet au profit de la réalisation d'une partie de cet objet (6).

Cet accès de l'ouvrier à la culture de son métier sera aussi l'occasion de l'éveil de sa conscience au rôle social qu'il devra désormais assumer dans une société où règne l'harmonie sociale. Dans ce contexte, l'esprit de la loi du 28 mars 1882 sur le travail manuel prenait en fait le contrepied de celui dans lequel avaient été créés les premiers ateliers annexés aux écoles primaires de garçons de la ville de Paris (7). La commission préparatoire à la loi du 28 mars 1882, présidée par A. Corbon et qui remit, en 1880, son rapport au Préfet de la Seine (8), devait freiner la création de ces ateliers professionnels, orienter le travail manuel vers une conception plus "éducative " au détriment de celle "économique " selon les propres termes de l'administration. Cette nouvelle orientation présentait également l'avantage de se démarquer nettement de l'enseignement privé confessionnel et/ou patronal et de le concurrencer sur le terrain de l'éducation technique élémentaire où son influence restait prépondérante (Mayeur, 1981, p. 262).

(6) Nous reviendrons dans la deuxième partie de cet article sur limportance de ce changement de méthode d'enseignement du travail manuel.

(7) L'école parisienne de la rue Tournefort, qui, depuis 1872. avait installé des ateliers de menuiserie. forge, ajustage et dont lenseignement pratique était suivi par l'ensemble des élèves. avait servi de modèle a lorganisation de cet enseignement professionnel primaire. A ce sujet, voir Laubier et Bourgueret. Le trarail mamuel à l'école de la rue Tourmefort. Paris. 1887.

En 1881. 100 ateliers étaient établis au- près de 175 écoles primaires de garçons de la ville de Paris (Exposition universelle de 1889. Rapports du jury international, publiés sous la direction d'Alfred Picard. Paris. Imprimerie Nationale. p. 3011.

(8) A Corbon. sénateur. Rapport à M. le Sénateur. Prefel de la Seine. sur le's exercices manuels dans les écoles primaires considerés comme complément de léducation. Paris. Imprimerie du Département de la Seine. 1880. La commission se compose. entre autres. de MM. A. Corbon. président désigné: A. Tolain. sénateur: $M$. Vadaud. député: D. Poulot et (;. Salicis. 
Par rapport aux premiers ateliers professionnels des écoles de la ville de Paris, l'atelier scolaire tel que le prévoit la loi du 28 mars 1882 est bien différent : tout semble organisé de façon à préserver l'élève du travail industriel et en particulier de sa pénibilité, qui n'est pas ici, contrairement aux formes d'apprentissage traditionnelles, saisie comme occasion pour l'apprenti de dépassement professionnel et personnel mais comme un obstacle à l'accès à une véritable culture manuelle.

\footnotetext{
"Quand ils se voyaient condamnés à corroyer une planche ou à dresser une pièce de fer pour débuter, les enfants de dix ans, mal renseignés. maladroits, s'épuisaient d'abord en efforts: puis, beaucoup prenaient le travail en dégoût et regardaient les camarades passer à dautres exercices tout en continuant eux-mêmes à corroyer mollement. " (9)
}

Cette conception de l'enseignement professionnel, qui s'enracine dans une vision fondamentalement émancipatrice de l'école républicaine, ne pouvait trouver défenseurs plus convaincus que chez ces anciens ouvriers d'élite comme A. Corbon, M. Nadaud, devenus sénateurs ou députés (cf. Offerlé, 1984) et qui adhèrent d'autant plus fortement à cette conception de l'enseignement professionnel que leur trajectoire professionnelle et sociale, les plaçant en représentants des ouvriers, leur interdit une réflexion critique sur le rôle qu'elle a joué dans la rupture avec leur culture d'origine. Parvenus au sommet de leur trajectoire sociale, "l'harmonie sociale" qu'ils souhaitent voir se réaliser n'est-elle pas l'état qui menace le moins leur position acquise, d'autant qu'elle justifie à leurs yeux le bien-fondé du rôle qu'ils ont joué et qu'ils auront à jouer auprès des classes ouvrières ? Ainsi s'explique, selon nous, la place très importante qu'occupe dans le rapport de $\mathrm{A}$. Corbon, à propos du travail manuel, le thème de "l'éducation harmonique" que l'école doit promouvoir. Dans ce rapport, l'enfant, qu'il s'agit d'éduquer et en particulier d'initier au travail manuel, est d'abord décrit comme être "naturel» dont les "trois ordres de faculté ", intellectuelle, morale et physique, ne sont pas séparés mais " étroitement solidaires". L'éducation doit tenir compte de cette réalité : "Oui, on s'est comporté jusqu'à ce jour dans l'enseignement (...) comme si la main, l'esprit, le cœur étaient insolidaires et conséquemment comme si on pouvait se dispenser de mener de front la culture des trois ordres de facultés". Cette description de l'enfant que nous donne le rapport de A. Corbon n'est autre que la métaphore d'un monde social où règnent l'harmonie, la solidarité entre les classes et les hommes, à laquelle aspire J. Ferry dans son discours de Vierzon et qui annonce le développement considérable du solidarisme. Elle est à rapprocher de la prolifération dès cette époque des associations de prévoyance et d'assistance de l'enfance en danger qui ont pour fonction, comme l'a montré F. Muel (1975, pp. 60-64), le contrôle et la moralisation de l'enfance dangereuse.

L'école qui a donné précocement à l'élève le goût et l'habitude du travail manuel évite la multiplication dans les grandes villes du "producteur

(9) R. Leblanc. Enseignement munuel et expérimental, 1, 35. Cité par Panthier. 1906, p. 76. 
immoral ", c'est-à-dire de l'ouvrier non qualifié, produit d'un exode rural récent et dont l'errance géographique et professionnelle représente une menace constante pour l'ordre social. Sur plusieurs points, l'enseignement du travail manuel pouvait se donner comme un moyen efficace de prévention sociale : en maintenant l'élève un peu plus longtemps à l'école, il le préservait de la perversion de la rue; en révélant ses aptitudes manuelles, il permettait à l'école d'accomplir sa mission en donnant à l'enfant des couches populaires une culture conforme à sa nature; en prolongeant l'ordre naturel, l'ordre social sera garanti puisque la variété des vocations et la diversité des tâches désignent les hommes à des fonctions sociales complémentaires. Le rapport de A. Corbon pousse plus loin encore le thème du rapprochement des classes et des hommes puisqu'il préconise, dès 1880 , la réunification de l'enseignement primaire " où les enfants de toute condition sociale commenceront obligatoirement leur éducation sur les bancs de l'école primaire ". Enfin, l'enfant est présenté comme un être dont la forme de l'intelligence est différente de celle de l'adulte et s'exprime à travers des besoins spécifiques d'" activité ", de "découverte", de "création". Le travail manuel lui convient parfaitement parce qu'il représente une matière concrète, non pas le concret donné dans sa matérialité, celle de l'outil ou du matériau, mais le concret apprécié pour sa valeur éducative parce qu'il convient à la forme d'intelligence de l'enfant.

La réalité du travail manuel dans les écoles primaires était bien différente de ce que pouvaient laisser supposer les positions officielles de l'administration. Une circulaire ministérielle du 28 octobre 1855 (10), soit trois ans et demi après l'institution de ce nouvel enseignement, reconnaissait qu'il n'était pas "vraiment sorti de l'Ecole normale " et qu'il devait être " particulièrement encouragé, sous peine de dépérir et disparaître bientôt "; elle chargeait $M$. Salicis (11), inspecteur général de travail manuel, de mener une enquête générale sur l'état du travail manuel dans les établissements scolaires. Les résultats de cette enquête amenèrent, en 1887, le ministre de l'Instruction publique à organiser, dans les écoles normales, pendant les vacances scolaires, des cours spéciaux de travail manuel à l'intention des instituteurs en exercice qui en feraient la demande. Ces cours, qui avaient une durée effective de 29 jours, étaient

(10) Bulletin administratif, $2^{c}$ semestre. tome xxxvilı. 1885.

(11) Gustave-Adolphe Salicis. né à l'île d'Aix (Charente-Inférieure) en 1818. sorti de l'Ecole polytechnique comme aspirant de marine. Il fut promu enseigne en 1842. lieutenant de vaisseau en 1848. En 1857, il quitta definitivement la mer pour devenir répétiteur de géodésie à l'Ecole polytechnique, avec le titre de capitaine de frégate. Républicain. s'intéressant aux questions d’économie sociale. il fut nommé délégué cantonal du $v^{c}$ arrondissement, ou il favorisa la création du premier atelier scolaire de l'école de la rue Tournefort. En 1882. il fut chargé par J. Ferry dune mission qui avait pour but d'étudier l'état de l'enseignement manuel en Allemagne et en Scandinavie. A son retour. il fut nommé inspecteur général de travail manuel. Il fonda, a Paris. la première Ecole normale de travail manuel. Il mourut en 1889, après avoir participé activement a lexposition universelle de 1889 et présidé la section technique du Congrès de lenseignement primaire. 
donnés aux élèves-maîtres par les professeurs des écoles normales chargés de l'enseignement du travail manuel (12). Il ne semble pas que ces cours fussent massivement fréquentés car une circulaire ministérielle prévoit un écourtement de leur durée, sous prétexte qu'elle éloignait un grand nombre d'instituteurs à qui elle imposait le sacrifice presque complet de leurs vacances (13) et une autre circulaire fait état de la décision prise par de nombreux conseils généraux qui, arguant du nombre limité d’inscriptions $\grave{a}$ ces cours, n'ont pas cru devoir voter les fonds nécessaires à leur organisation (14). Prenant acte de ces échecs successifs, l'administration supprima définitivement ces cours en 1889 (15). Les statistiques officielles établies pour l'exposition universelle de 1889 nous fournissent un autre indicateur des difficultés que rencontra l'administration pour généraliser cet enseignement. En effet, elles évaluent à 12000 le nombre d'écoles élémentaires où les rudiments du travail manuel avec ou sans atelier sont enseignés et à 258 seulement le nombre de celles pourvues d'ateliers, dont 103 sont situées dans le seul département de la Seine (16). Enfin, le jury international de l'exposition universelle de 1900 constate dans son rapport final que, "sauf à Paris où la méthode a pris corps (...), l'enseignement manuel est resté stationnaire; il a même décliné dans beaucoup d'écoles élémentaires ". Après avoir dégagé les avantages du travail manuel, le jury conclut : "C'est ce que n'ont pas compris malheureusement les maîtres qui ont trouvé plus facile de tourner en ridicule le travail scolaire que d'en faire un essai loyal, que d'en étudier la portée pédagogique " (17). Le recensement de tous ces faits semble indiquer que dans leur grande majorité les instituteurs n'avaient pas suivi les innovateurs. Le travail manuel avec atelier restait un phénomène minoritaire, essentiellement urbain, plus particulièrement parisien; il n'était pas parvenu à s'implanter dans l'ensemble des écoles primaires, alors que son enseignement était rendu obligatoire depuis la loi du 28 mars 1882.

Dans ce contexte, le Congrès du Havre de 1885 avait pour fonction, par l'intermédiaire des inspecteurs primaires fortement représentés et des instituteurs acquis aux vues de l'administration, de donner l'impulsion nécessaire à l'application de cet enseignement, mais sous forme adaptée qui tienne compte des difficultés rencontrées à le généraliser. C'est ainsi qu'était réaffirmé le refus de faire de cet enseignement un premier cycle d'enseignement professionnel pour lui préférer celui d'une culture ma-

(12) Circulaire ministérielle du 21 mars 1887. Circulaires el instmations officielles. lome 10, p. 91.

(13) Circulaire ministérielle du 22 mars 1888. Circulaires et instructions officielles. tome 10, p. 258.

(14) Circulaire ministérielle du 10 juillet 1888. Circulaires ef instructions officielles. tome 10 , p. 312 .

(15) Voir à ce sujet G. Salicis. Enseigne- ment du travail manuel, mémoires et documents scolaires publiés par le Musée Pédagogique, fasc. $n^{\prime \prime} 33$, Paris, Imprimerie Nationale, 1889.

(16) Exposition universelle de 1889. Rapport du jury international, op. cit.. p. 298.

(17) Exposition universelle de 1900. Rapports du jury international. Groupe I "Education et enseignement", Paris, Imprimerie Nationale, p. 992. 
nuelle générale et qu'était avancée l'idée d'un travail manuel éducatif dont l'enseignement n'exigeait pas forcément un travail en atelier. Les organisateurs du Congrès du Havre réalisaient en quelque sorte un compromis : tout en conservant l'idée de son initiateur, G. Salicis, d'un travail manuel exercé en atelier, ils tentaient, en présentant le travail manuel " comme le complément de l'enseignement primaire "(18), de concilier les positions des instituteurs en proposant également une conception plus scolaire du travail manuel (19).

\section{II. - Le travail manuel à l'école primaire : d'une culture manuelle générale au travail manuel éducatif}

Ce n'est pas le moindre paradoxe de cette étude que de constater que les instituteurs participant au Congrès du Havre présentent la question du travail manuel à l'école primaire en déplorant que celle-ci, qui se définit comme "école du peuple ", dispense un savoir trop livresque, ne prépare pas ses élèves à leur futur état de travailleur manuel et les éloigne des professions manuelles. Ces critiques du contenu de l'enseignement primaire, l'exhortation à l'exercice du travail manuel dès l'école primaire, l'exaltation de ses vertus sonnent étrangement sous la plume de ces promus, pour la plupart fils d'agriculteurs, d'artisans, d'ouvriers qualifiés, pour qui l'enseignement primaire a eu pour principale fonction de les détourner de l'exercice de professions manuelles (Muel, 1977). Cette situation ne les conduit pas à douter de la réussite de cette innovation pédagogique en se prononçant, par exemple, contre l'introduction du travail manuel à l'école primaire, ou en marquant nettement les limites de ce nouvel enseignement (20); au contraire, tout se passe comme si cette première rupture avec leur culture d'origine qu'a représentée leur entrée dans le métier d'instituteur les portait à concevoir l'enseignement du travail manuel à l'école primaire comme la chance des enfants des classes populaires, comme le moyen qui favorisera leur libération après avoir permis la leur en les faisant accéder à la dignité culturelle.

(18) C"est dans ces termes qu était formulée la question traitant du travail manuel au Congrès du Havre.

(19) Cette nouvelle orientation arait déjá été amorcée quelques mois auparavant par la suppression de l'Ecole normale spéciale de travail manuel de la rue Thuilier et lintégration par décret du 4 septembre 1884 (Bulletin administratif. tome $\times \times \times v$. Paris, 1884) de son enseignement dans les programmes des élèves-maîtres de l'Ecole normale supérieure de Saint-Cloud. Les difficultés ne tardèrent pas à surgir entre loorientation donnée par G. Salicis à cet enseignement et le choix des travaux proposés aux élèves- maîtres. ou les exercices de pliage et de cartonnage étaient nettement préférés a ceux datelier. Ainsi, en perdant l“indépendance du recrutement et de la formation de ses maitres, le travail manuel à l'école primaire avait perdu du même coup sa finalité propre.

(20) Seulement deux mémoires sur quarante-huit se prononcent contre lintroduction du travail manuel à lécole primaire. Il faut signaler cependant que les participants au Congrès du Havre neétaient pas délégués par lensemble de leurs collègues. ce qui explique certainement quils aient été si nombreux à être daccord avec les positions de ladministration. 
Il reste maintenant à analyser comment cet enseignement va être pensé pour permettre aux enfants des couches populaires d'atteindre un but aussi ambitieux.

Ce souhait de préparer leurs élèves à leur futur état de travailleur manuel amène les instituteurs à préconiser d'étayer l'enseignement du travail manuel sur les demandes utilitaristes de leurs familles, en conseillant la fabrication dans l'atelier scolaire d'objets complets ayant une utilité pratique, comme les ustensiles de ménage, les outils aratoires ou de jardinage. Ce souci d'organiser un enseignement pratique ne les conduit pas cependant à souhaiter la création d'un enseignement réellement productif. Les objets fabriqués dans l'atelier scolaire ne doivent présenter aucune valeur marchande, sous peine de "subordonner à des intérêts matériels des avantages intellectuels et moraux bien supérieurs " (directeur d'école primaire de Nancy).

Comme nous l'avons dit précédemment, cet enseignement n'a pas pour but de constituer un premier degré d'enseignement professionnel, mais de développer chez l'élève l'habileté manuelle à tous les métiers en favorisant l'éducation de l'œil, de la main, des sens en général, en exerçant précocement des qualités comme l'attention, l'observation, la précision et en donnant le goût du travail.

"Quelles professions allez-vous apprendre aux élèves? A cela nous répondons que notre but n'est pas denseigner une profession spéciale (...). Il s'agit seulement de leur inspirer le goût du travail, de leur rendre la main habile a manier les instruments, de développer chez eux l'esprit d'observation. de les préparer en un mot à une profession quelconque en leur donnant des notions générales. " (directeur d’école primaire à Nîmes) (21)

C'est d'abord parce qu'elle rappelle les réalités de l'atelier industriel caractérisé par la division du travail que la spécialisation professionnelle est décrite comme "réductrice » et "aliénante » et, à ce titre, catégoriquement refusée par les instituteurs. C'est ensuite parce qu'elle est le plus souvent imposée par les parents et l'employeur ou laissée au hasard des circonstances que les instituteurs la rejettent. Elle s'oppose trop à leur représentation du choix professionnel qui, par référence à la manière dont ils sont entrés dans le métier, ne peut être que l'expression d'une vocation. A cet effet, les instituteurs proposent de multiplier les situations manuelles pour "surprendre", "provoquer " le don de l'élève. Un instituteur de

(21) Ferdinand Buisson (1888, pp. 18241825), citant la Revue de l'enseignement professionnel. formulait le probleme en des termes identiques: "De même que dans le monde physique un petit nombre de lois expliquent une infinité de phénomènes (...). le travail industriel peut être rapporté à quelques opérations fondamentales. telles que dresser ou aplanir. ajuster, tourner. etc. Les matériaux divers sur lesquels on opère se classent à leur tour par grandes catégories, supposant des procédés de travail analogues... Les principaux types doutils se rapportent a ces propriétés diverses des matériaux. et on peut les ranger de même en un petit nombre de groupes ". Sil en est ainsi, il est permis de conclure à la possibilité d’un enseignement manuel diun caractère général, donnant à tous les élèves des connaissances théoriques et une habileté pratique qui nexige plus ensuite. pour sappliquer à tel métier particulier. qu un court apprentissage. 
Marseille trouve, à ce sujet, des accents d'une modernité pédagogique pour exposer la démarche qu'il préconise pour découvrir chez l'élève tous ces talents cachés.

"Ce n'est pas assez que lenfant voie. Il est bon qu'il participe de ses petites mains a la peine du travail sans apparence de contrainte. presque de lui-même et à sa fantaisie. Souvent il sera déçu. Son inexpérience ly condamne. Il sessayera à plusieurs tâches (...). Apres bien d hésitation. lenfant śadonnera avec une préférence marquée et constante a une occupation bien en rapport avec ses moyens. Ainsi se révèle le goût naissant et délicat. Gardons-nous de l'étouffer sous une réglementation trop étroite. Bornons-nous à en favoriser largement le libre exercice."

Le respect des personnes et de leur vocation reste cependant formel car il n'est pas rapporté à une reconnaissance des particularités de métier. Toute spécificité professionnelle est négligée tant au niveau du matériau à travailler que de l'outil à utiliser qui ne sont jamais adaptés à l'opération à exécuter, à l'ouvrier qui s'en sert (22).

"Nest-il pas vrai que lhabitude à manier un outil rend apte au maniement des autres?" (directeur d'école primaire à Nimes)

Cette méconnaissance du caractère unique de l'outil conduit les instituteurs à le réduire à un simple instrument de moralisation des élèves, au mépris de sa fonction première d’instrument de l’acte technique.

"Consultons dabord le programme et nous verrons que c'est surtout l'habitude de lordre et du travail, la rectitude et la promptitude dexecution quon doit chercher à inculquer aux enfants de 6 a 11 ans. " (instituteur a Sigy-en-Bray. Seine-Inféricure)

En conséquence, les instituteurs tendent à utiliser indifféremment les types de matériaux et d'outils, comme cet instituteur de Paris qui, sous prétexte d'économie, préconise de remplacer le bois par le carton et la scie par une paire de ciseaux et affirme en avoir fait l'expérience et avoir obtenu, dans ces conditions, de "bien meilleurs résultats". Pour cet instituteur, la démarche utilisée est privilégiée aux dépens du résultat obtenu, et son ignorance totale de la spécificité du matériau, de l'outil et des résistances qu'ils peuvent opposer à l'apprenti dans l'exercice de son métier a pour résultat qu'il ne doute pas de la réussite du transfert d’apprentissage du matériel pédagogique au matériau professionnel.

De la même façon, l'enseignement des formes de graphismes techniques spécifiques aux différents métiers, comme le trait par exemple, sera délaissé au profit exclusif de l'enseignement du dessin qui, apprécié pour son caractère d'universalité, est présenté dans tous les mémoires comme la "base du travail manuel".

(22) "Il (l'outil) est unique. il est difficilement transmissible au-delà du geste stéréotypé et de la pensée qui est censée lexpliquer. Il est tributaire de la main qui le manie. de l'idée qu'on se fait de son usage. Il est fait dune manière particuliere. il a une forme adéquate: son poids. son équilibre. son manche sont les liaisons entre lui et la main. Et tout cela au point que louvrier. jadis. personnalisait ses outils. les finissait. les adaptait a sa façon de travailler." (Gille. 1978. pp. 1426-1427) 


\section{Revue française de sociologie}

"Le travail manuel est intimement lié au dessin. Celui-ci est au travail ce que l'écriture est à la pensée. il en est la traduction, il fixe les idées, il les représente (...). c'est-à-dire quavant de passer au travail manuel, nous devons familiariser nos élèves avec le dessin. " (instituteur à Saint-Dizier)

Cet extrait de mémoire situe le dessin du côté de la traduction des idées, de la représentation: apprendre un métier, c'est d'abord apprendre un langage et le système des signes qu'il comporte. Pour cet instituteur, le dessin représente le véritable "outil» du travailleur manuel, celui qu'il doit parfaitement maîtriser, parce qu'il permet le passage de l'esprit entre la réalité et la représentation et qu'il ménage toutes les transitions entre le concret et l'abstrait. Enseignée dans cet esprit, la connaissance du dessin fonde la dignité ouvrière en distinguant le «bon ouvrier" du "simple exécutant ", reprenant en cela l'esprit des écoles de dessin du XvII" siècle (23). L'ordre de succession des apprentissages reproduisant l'ordre d'exposition des connaissances, l'élève devra apprendre à dessiner l'objet avant de le fabriquer, tous les mémoires insistent longuement sur ce point. On peut s'interroger sur l'efficacité professionnelle de cette succession d'apprentissages érigée par les instituteurs en principe pédagogique : si le dessin est nécessaire à l'ouvrier qualifié au moment de la conception de son travail, il ne va pas de soi que l'apprenti doive d'abord dessiner l'objet qu'il ne sait pas encore réaliser, le dessin de l'objet ne le rendant pas automatiquement capable de le fabriquer (24). Dans d'autres formes d'apprentissage professionnel qui ne sont pas scolaires, on préférera confronter tout de suite l'apprenti à la fabrication de l'objet, celui-ci n'étant dessiné qu'après avoir été réalisé. Le dessin prend alors une tout autre signification : réintroduit comme étape d'une démarche plus spécifiquement manuelle, il n'est plus le support d'un savoir abstrait, mais ce qui permet à l'apprenti d'accéder à la compréhension des difficultés qu'il a rencontrées dans l'exécution de son travail.

Cette conception du travail manuel interdit également tout recours aux recettes, astuces techniques, procédés, particuliers aux différents métiers.

«Cet enseignement (du travail manuel) aurait pour but d’abord d’éclairer les élèves sur la routine. les préjugés. les pratiques vicieuses et depuis longtemps condamnées

(23) "Les pédagogues du XVIII siècle en France avaient commencé à comprendre limportance de l'enseignement du dessin. Ils exagéraient même sa valeur : 'Le dessin est la base de tous les ouvrages mécaniques'. dit un rapport conservé dans les archives navales, et Les ouvriers ne sont supérieurs les uns aux autres qu'autant qu'ils sont plus ou moins experts dans cet art'. " (cité par Artz. 1938). "Cette déconnexion d'avec le métier. présentée ici comme l'un des avantages du dessin qui, dans son universalité, convient à tous. sera par la suite une des tares des écoles de dessin, ou du moins une des difficultés à surmonter quand le public sera composé en majorité par les ouvriers de lindustrie mécanique et non plus par les représentants mal définis des arts mécaniques. " (Deforge. 1981, p. 168)

(24) "Dessiner les plans d'une machine n'est pas la fabriquer: faire le schéma d'un circuit électrique n'est pas le câbler. Ce que l'action sur le monde symbolique ne reflète pas de l'action sur le monde matériel et social, ce sont d'abord les effets non voulus qu'entraine la résistance du réel. " (Malglaive et Weber, p. 26) 
qui sont encore en usage dans la localité. de substituer des idées saines. de meilleurs procédés, ceux sanctionnés par la science el lexpérience. " (instituteur à SaintNicolas-de-la-Taille. Seine-Inférieure )

L'opposition entre "pratiques" et «idées", qualifiées respectivement de "vicieuses" et "saines", révèle que pour cet instituteur le travail manuel ne peut recevoir sa légitimité que des principes scientifiques qui le fondent. "L'expérience " n’est plus ici l’aboutissement d'un savoir-faire acquis dans la pratique professionnelle, mais se définit comme l'expérimentation de la démarche scientifique (cf. Malglaive et Weber, 1982, p. 21 ). De la même façon, cet équilibre entre la main et l'esprit, que tant d'instituteurs souhaitent réaliser à travers cet enseignement, n'est plus celui atteint dans la pratique professionnelle par le jugé, l'imitation, le tâtonnement, l'essai et l'erreur, mais celui qu'acquiert une main désormais maîtrisée par la connaissance des "principes raisonnés" du métier. Un extrait de mémoire d'un instituteur de Meurthe-et-Moselle résume la logique des discours qui définissent la finalité du travail manuel dans l'atelier scolaire :

"Il y aura une grande différence entre le travail manuel dun atelier scolaire et celui dune manufacture: dans une manufacture. tout est réduit à une simple et abrutissante routine: dans latelier scolaire. tout le travail scolaire est fait en vue de louvrier. Ce n'est pas l'objet qu'on lui fait fabriquer qui est important. cest lui-même."

Les participants au Congrès de l'enseignement primaire et de l'enseignement primaire supérieur qui se tient à Paris en 1889 , poussant plus loin encore l'orientation donnée par le Congrès du Havre, décidèrent "qu'en France, lenseignement industriel est incompatible avec le programme des écoles primaires et des écoles normales " (25). Cette résolution aboutit à la réforme du 3 juin 1891 qui institua le travail manuel sans atelier : les exercices de pliage, de découpage, de cartonnage prirent la première place des programmes et furent exécutés par tous les élèves, même par ceux des cours supérieurs. Les ateliers ne furent pas supprimés mais leur programme fut modifié: les travaux de tour furent supprimés, ceux d'ajustage remplacés par des exercices en fil de fer demi-rond, enfin les travaux de lime et de burin ne furent abordés qu'au cours supérieur par les élèves âgés de 12 ans. C"est dans cet esprit qu'on peut également comprendre, comme nous l'avons déjà vu, l'abandon dès 1887 dans les écoles normales de la méthode d'enseignement du travail manuel des "objets utiles" et son remplacement par celle des "éléments techniques " qui, à la différence de la première, n'exigeait plus la fabrication d'un objet complet. On pourra, à travers le témoignage d'un adversaire de cette deuxième méthode, se faire une idée de la manière dont elle était conçue, même si la description qu'il en donne reste partiale.

(25) ('f. le rapport de E. Monod dans Exposition universelle de 1889. 1. I. 1890. p. 318. 


\section{Revue française de sociologie}

"Ce qu'il nous est possible de constater. c'est l'état de l'enseignement dans les écoles primaires de Paris, dont 92 sont dotées de loutillage pour le travail du bois. La, point d'objets d'un usage commun à confectionner, mais des éléments détachés, des planchettes de $0.30 \mathrm{~m}$ de longueur sur $0.10 \mathrm{~m}$ de largeur, sur lesquelles l'enfant s'exerce et qui après complet achèvement prennent le chemin du poêle. C'est là un résultat qui n'intéresse que médiocrement l'élève et qui n'est pour lui d’aucune utilité personnelle; j'ajouterai que c'est un procédé peu propre à inspirer aux enfants des idées d’économie. Ces pièces de bois qu'il corroie, qu'il met d'équerre. sur lesquelles il promène la scie. dans lesquelles il creuse des mortaises. présentent une uniformité désespérante et ne peuvent point donner lieu a la solution de problèmes utiles concernant l'histoire naturelle. la géométrie. le système métrique. " (E. Schmitt. directeur d'école communale à Paris) (26)

Sous l'impulsion des inspecteurs de travail manuel R. Leblanc et $\mathrm{A}$. Jully, cette deuxième méthode fut présentée à l'exposition universelle de 1900 comme supérieure à la première parce que plus étroitement reliée à l'enseignement du dessin géométrique. Le travail manuel devenait en quelque sorte une matière auxiliaire de celle du dessin et, en dissociant le dessin de la réalisation d'un objet complet, la nouvelle méthode tendait à faire du dessin un enseignement autonome ayant sa finalité propre.

«Dans les ateliers. on admet que tout travail bien tracé est à moitié fait. La conséquence. c'est quón devra s'occuper d'abord et surtout de la rigueur du tracé. » (Panthier. 1906. p. 184)

Le travail manuel devenait ainsi, encore plus nettement que lors du Congrès du Havre, un moyen pédagogique, en apportant à l'enseignement des matières abstraites une application concrète. A l'image des manipulations de l'enseignement secondaire, les exercices proposés étaient des applications, des vérifications, des démonstrations de notions de dessin, de géométrie, de physique étudiées en classe ou, servant de point de départ à la découverte de ces notions, ils s'apparentaient plus nettement à la leçon de choses dont l'enseignement se développait à cette époque (Calderon, 1977). Il était alors conçu, en dehors de toute visée professionnelle, comme le support concret qui permettait à des enfants jugés rebelles à l'enseignement abstrait d’accéder à la compréhension de notions théoriques.

"Comme conséquence. la culture de l'ail el de la main est négligée: or le travail manuel éducatif, intimement relié au dessin. est surtout destiné à combler cette lacune et du même coup à contrebalancer laction trop exclusivement intellectuelle de lancien système pédagogique. C'est un adjuvant à l'enseignement ordinaire qu’il facilite en apportant le concret a côté de l'abstrait: c'est un des premiers facteurs de l'éducation des sens, notamment de loeil et de la main, organes indispensables a l'ouvrier pour gagner sa vie. " (27)

En résumé, dissocié dès l'origine de la réalité des métiers et de leur spécificité, le travail manuel pouvait être réinterprété de façon scolaire pour devenir le travail manuel éducatif. Il perdait ainsi tout caractère professionnel, au point que les directeurs d'écoles pratiques de commerce

(26) Revue pédagogique, premier semestre 1887. p. 148
(27) Exposition universelle de 1900. op. cit., p. 992. 
et d'industrie en déconseillèrent l'exercice aux élèves qui se destinaient à rentrer dans les écoles qu'ils dirigeaient (28).

\section{III. - Le travail manuel à l'école primaire : un enseignement impossible?}

Il reste à tenter de comprendre pourquoi les instituteurs sont restés hostiles à l'introduction du travail manuel à l'école primaire; s’ils pouvaient douter que cet enseignement constituât la chance du monde ouvrier, encore pouvaient-ils espérer qu'elle fût celle de l'école. En s'adaptant aux caractéristiques des enfants appartenant aux couches populaires, en reprenant les demandes utilitaristes de leurs familles en matière d'éducation, le travail manuel pouvait effectivement se donner comme un moyen de réconcilier les enfants des classes populaires avec l'école, de la rendre plus intéressante et efficace, en limitant l'absentéisme scolaire, ou au contraire en évitant que toute scolarité réussie conduise le fils d'ouvrier à quitter son milieu d'origine. Les instituteurs auraient pu espérer trouver dans le travail manuel la réponse aux problèmes que leur posait l'obligation scolaire, en quelque sorte le remède à la crise de l'enseignement élémentaire.

"Voyez avec quelle satisfaction les élèves les plus rebelles à nos leçons quittent l'école pour aller se livrer aux travaux manuels. Répétez-leur tant qu'il vous plaira que l'instruction est le plus précieux des trésors, vous ne tarderez pas à remarquer que vous parlez à des sourds pour la raison que l'enseignement de l'école n’a pu réussir à les intéresser. " (instituteur à Clipponville, Seine-Inférieure)

Mais penser qu'il soit possible pour les instituteurs d'utiliser le travail manuel comme moyen de modifier le rapport que les enfants des couches populaires entretiennent avec le savoir scolaire serait oublier que la pratique pédagogique des instituteurs est marquée par le rapport aux couches populaires qu'ils doivent à leur origine ou à leur trajectoire sociale. Il n'est pas facile de retrouver les chemins du travail manuel quand toute la scolarité du bon élève de l'école primaire puis de l'Ecole normale qu'a été celle de l'instituteur a consisté justement à l'en détourner (Molinari, 1975). Les instituteurs qui adhèrent à ce projet d'introduire le travail manuel avec atelier proposent, comme nous l'avons vu, un enseignement de culture manuelle générale. Celui-ci s'oppose en fait à un enseignement qui, en s'enracinant dans la réalité des cultures de métier, serait conçu dans le prolongement de la préformation culturelle des

(28) "Beaucoup de directeurs (...) nous affirmaient qu'avec ces exercices de pliage. de cartonnage et de découpage ordonnés par les programmes, on n'obtenait aucun résultat sérieux. Plusieurs directeurs d’écoles pratiques prétendaient même qu'il était préférable que les élèves qui leur arrivaient des écoles élémentaires, pour faire leurs études professionnelles. n'eussent jamais été exercés à ces travaux puérils qui leur font contracter des habitudes défectueuses car plus tard ils ont beaucoup de peine à s'en défaire. "(Paquier. 1908, p. 108) 


\section{Revue française de sociologie}

enfants des classes populaires (Testanière, 1982). Cette position qu'ils défendent au Congrès du Havre en accord avec celle de l'administration et qui, nous l'avons vu, ne sera pas adoptée par la majorité des instituteurs ne peut se comprendre que si on la rapporte à leur trajectoire professionnelle. Une analyse de 50 fiches individuelles de carrière professionnelle des instituteurs de l'arrondissement du Havre ayant participé au Congrès du Havre, comparée à celle d'un groupe témoin de 86 instituteurs des mêmes cantons n'y ayant pas participé (29), permet d'apercevoir que, s'il est vrai que les premiers sont plus âgés (40\% ont 40 ans et plus) que les seconds $(27,90 \%)$, ce qui pourrait pour une part expliquer qu'ils aient été plus mobiles géographiquement ( $58 \%$ contre $45,34 \%)$, ils sont mieux intégrés à l'école puisque plus souvent normaliens $(62 \%$ contre $33,72 \%)$ et ont réussi leur carrière au point d'exercer dans une ville petite ou grande $(64 \%$ contre $47,67 \%)$. Si on veut résumer la logique de la trajectoire professionnelle de ces deux groupes, on pourrait opposer à un premier type d'instituteur, normalien ayant réussi à rejoindre un bourg, puis après deux changements de postes au moins la grande ville, un deuxième type d'instituteur, plus jeune sans doute mais non normalien et qui continue d'exercer son métier dans le premier poste de campagne où il a été titularisé. Ainsi, il n'est pas interdit de penser que les instituteurs participant au Congrès du Havre seraient d'autant plus enclins à transformer l'enseignement des métiers en un enseignement de culture générale que leur trajectoire est ascendante, par là peut-être que leur volonté de libérer les enfants des couches populaires des servitudes du métier manuel correspondrait à la manière dont ils ont su se libérer de la condition ordinaire du métier d'instituteur; leur participation au Congrès du Havre manifesterait en quelque sorte la réussite de leur carrière (30).

Quant à la majorité des instituteurs, qui n’appliqueront pas les résolutions votées au Congrès du Havre et dont les caractéristiques doivent être proches de celles de notre groupe témoin, on peut avancer l'hypothèse qu'ils ne peuvent adhérer à cette transformation du travail manuel parce qu'ils ne peuvent s'arracher complètement à leur origine, c'est-à-dire renier le travail du père et les sacrifices consentis pour les avoir menés là où ils sont parvenus. En un mot, on peut penser qu'ils ne peuvent adhérer à cette conception du travail manuel car ce serait en définitive se séparer d'eux-mêmes.

Pour que le travail manuel soit conçu dans le prolongement de la préfiguration culturelle des enfants des classes populaires, il aurait fallu, semble-t-il, que son enseignement échappât aux instituteurs pour être donné par des ouvriers, suivant le principe de l'alternance entre le travail

(29) Tous ces documents ont été déposés aux archives départementales de RouenCôte. 7 TP.

(30) Il est peut-être paradoxal de suggérer que ceux qui ont eu la meilleure réussite scolaire, au lieu de tenir pour lordre qui leur a permis cette réussite. soient favorables à l'innovation. Les documents manquent, mais peut-être ceux qui ont le mieux réussi ont-ils été. et sont-ils encore alors, ceux qui se rallient le plus volontiers a l'innovation préconisée par la hiérarchie administrative. 
dans l'atelier industriel et son enseignement dans l'atelier-école. Mais ce serait méconnaitre le fonctionnement du système scolaire que de penser qu'il puisse prendre en compte une telle mesure : en dissociant le savoir professionnel et la fonction éducative de celui qui dirigerait les travaux manuels, argument décisif pour écarter le recrutement d'enseignantsouvriers, les instituteurs réunis en congrès au Havre n'adoptaient-ils pas le point de vue qui sauvegardait le mieux, en définitive, leur position acquise? Le travail manuel ne pouvait être enseigné que par un homme formé au métier d'éducateur, c'est-à-dire formé à la transmission des savoirs, et cette qualité, selon eux, fait défaut à l'ouvrier.

"Pénétrons-nous surtout de cette vérité. c’est qu'il s'agit moins de savoir que de savoir communiquer. Cette dernière qualité, nous la reconnaissons à l'instituteur et nous la nions à l'ouvrier qui viendrait apres la classe diriger les exercices de l'atelier scolaire. " (directeur d’école primaire à Paris)

A l'ouvrier, il est d'abord reproché son manque de connaissances théoriques; c'est ainsi que dans les écoles normales son rôle auprès des élèves-maîtres se limite à la préparation des matières premières et à l'entretien de l'outillage. Toute la direction de l'enseignement incombe au professeur de travail manuel, comme le souligne l'inspecteur de travail manuel R. Leblanc (1895, p. 36) :

"Louvrier ne se rend pas compte en général des raisons scientifiques des différentes opérations qu'il exécute, le professeur doit donner ces raisons, (...) il doit expliquer les motifs qui ont fait adopter la forme. la disposition, la nature des différentes pièces du matériel en usage; en un mot, il doit tout expliquer et donner la raison scientifique de ses explications. »

A l'ouvrier, il est aussi reproché ses difficultés à faire respecter l'ordre scolaire :

"Aussi, dans la plupart des cas, a-t-on dû se contenter d'un recrutement local, en s'adressant ordinairement à de bons ouvriers (...) dépourvus des talents d'enseigner, souvent même n'ayant, faute d’éducation suffisante. que peu d'autorité sur les élèves." (Fouqué, 1900, p. 124)

On trouvera dans la description que donne A. Panthier de l'enseignement manuel suédois les arguments décisifs qui ont amené l'administration à écarter les ouvriers de l'enseignement du travail manuel. Cette description est importante pour notre étude si l'on sait que le système suédois servit de modèle à l'organisation du travail manuel français (Salomon, s.d.).

"Jusque-là on avait pris les maîtres partout, dans l'enseignement et dans les usines, même dans les armées (...). La cause principale des échecs précédents est facile à expliquer. On a complètement négligé le côté pédagogique en chargeant de cet enseignement des artisans, des hommes de métier. Ceux-ci ne pouvaient s'acquitter de leur tâche quen artisans. Accoutumés à ne rien voir au-delà de leur métier, ils considéraient l'école comme un atelier et les enfants comme des apprentis dont il fallait faire d'habiles ouvriers. Or cela est contraire aux principes de la pédagogie classique (...). Pour quine instruction quelconque devienne utile, il faut quelle contribue à l'éducation (...). C'est précisément la capacité d'associer d'une manière intime et raisonnée ces deux fonctions. instruire et élever. qui distingue l’instituteur de l'artisan. 


\section{Revue française de sociologie}

Ce dernier ne s'occupera que de la partie technique et négligera entièrement l'éducation. Chaque fois que, dans une école, on néglige l'éducation, l'établissement ne tardera pas à péricliter. " (Panthier, 1906, pp. 97-98)

Pour mieux évaluer cette capacité à l'enseignement, l'administration, par l'arrêté du 3 janvier 1891, modifia les conditions de l'examen du certificat d'aptitude à l'enseignement manuel de façon à permettre au jury de juger les candidats " non seulement sur leur habileté manuelle mais sur leur aptitude à l'enseignement du travail manuel " (Leblanc, 1895, p. 13). L'enseignement du travail manuel devait respecter certaines règles pédagogiques, sous peine de perdre toute valeur éducative : la méthode suivie devait être "rationnelle", il fallait décomposer l'exercice en différentes opérations, les analyser, en donner les raisons scientifiques, les graduer selon un plan méthodique qui tienne compte de l'âge de l'enfant et de son développement intellectuel. Cette dissociation entre le savoir et sa transmission qui naît avec la scolarisation des apprentissages va donc trouver, avec la reconnaissance d'une spécificité de l'enfance et des étapes de son développement, un fondement scientifique qui permettra la légitimation d'un corps de professionnels de l'éducation technique dont le savoir, parce qu'il ne se réduit pas seulement à la somme des connaissances professionnelles, mais aussi à la manière de les enseigner, devient indépendant de l'exercice du métier manuel (31).

A partir des années 1890 , le travail manuel deviendra pour une minorité d'élèves fréquentant certaines écoles urbaines une préparation à l'Ecole pratique de commerce et d'industrie (32) et, pour une majorité d'entre eux, des activités manuelles éducatives. Ainsi, paradoxalement, le travail manuel dont l'introduction à l'école primaire avait été présentée comme le remède à la crise de l'apprentissage n'aura pas comme finalité première de préparer la majorité des élèves à devenir des ouvriers. On touche là une des contradictions fondamentales de l'école dont $R$. Leblanc, inspecteur de travail manuel, nous donne une illustration, en décrivant l'attitude des élèves devant un atelier de forge.

"Quand les élèves à côté de cette lueur d'espoir, la vie bourgeoise, verront cet enfer, la forge, ce salaire insuffisant, péniblement acquis, ces vêtements grossiers, ces mains noires, combien consentiront à être ouvriers? Tout ce qu'il y a de noble dans la profession d'ouvrier, tout ce que la démocratie peut assurer, après quelques années de persistants efforts, à l'artisan économe et laborieux, disparaîtra devant leurs yeux. " (33)

Dans ce sens, on peut faire l'hypothèse que le travail manuel à l'école primaire, qui aurait pu constituer un premier degré d'enseignement professionnel, a été, si l'on peut dire, abandonné aux classes de perfectionnement, qui se créèrent à la même époque dans les grandes villes et dont

(31) On sait ce qu'il advint des maîtresouvriers de la première école d'apprentissage fondée au Havre en 1867, qui furent progressivement intégrés au corps professionnel des instituteurs. A ce sujet, cf. Rougier-Pintiaux, 1983, p. 660.
(32) Cf. à ce sujet le succès des enseignements spéciaux des écoles primaires de la ville de Paris.

(33) R. Leblanc, Mémoires et documents, 46, 109, cité par Panthier, 1906, p. 76. 
il allait, par opposition à l'enseignement normal, constituer l'enseignement spécifique en proposant un enseignement concret qui convenait bien à des "intelligences concrètes". "Le principal procédé d'éducation qu'on emploie et qui amène ces résultats, c'est le travail manuel (...). En même temps, au point de vue intellectuel, les idées qui ne leur entrent pas dans la tête par les abstractions que leur servent les livres, leur entrent par les doigts, par l'appareil musculaire, pourrais-je dire, grâce au travail manuel. " (34)

C'est dans ce contexte qu'il faut comprendre le thème choisi pour le premier examen du certificat d'aptitude à l'enseignement des enfants arriérés: "L'importance du travail manuel dans l'éducation des arriérés " (35). De cette façon, tout risque de concurrence entre les spécialistes de l'enseignement primaire normal et spécial était écarté, la distinction entre ces deux types d'enseignement respectait la hiérarchie des savoirs et l'enseignement spécial pouvait se donner comme un moyen plus efficace de remédier à la crise de l'enseignement élémentaire en contrôlant plus étroitement les enfants des classes populaires.

Ainsi l'école primaire n'a-t-elle pu concevoir un enseignement manuel, à l'intention des enfants des classes populaires, qu'en le transformant, en le dissociant dès l'origine des métiers et de leurs particularités; il a d'abord été conçu comme un moyen de culture manuelle générale puis il a été ensuite réinterprété, de façon plus scolaire encore, comme travail manuel éducatif.

Dans cette mesure, apparaît l'incapacité de l'école traditionnelle à enraciner son enseignement dans les réalités des cultures de métier, par là à prendre en compte le milieu culturel des enfants des classes populaires traditionnelles. Pour que le travail manuel pût réconcilier les enfants de ces milieux avec l'école, peut-être aurait-il fallu que son enseignement ne fût pas confié aux instituteurs mais à des ouvriers en alternant l'exercice du travail dans l'atelier industriel et l'enseignement dans l'atelier-école. Ainsi, semble-t-il, aurait-il conservé son caractère professionnel et ouvrier. Si le travail manuel n'a pu être intégré dans les programmes qu'après avoir été coupé des pratiques sociales du travail, retravaillé selon les principes de la pédagogie, c'est peut-être qu'il ne pouvait être reconnu par l'école qu'en étendant la polyvalence de l'instituteur comme professionnel de la transmission de tout savoir.

Pascale ROUGIER-PINTIAUX

UFR de psychologie, sociologie et sciences de l'éducation Université de Rouen, rue Lavoisier. 76130 Mont-Saint-Aignan

(34) Discussion du projet de loi ayant pour objet la création des classes de perfectionnement, Journal officiel. 1909. Sénat. débats parlementaires, p. 103.

(35) Revue pédagogique, 1910, $2^{c}$ semes- 


\section{RÉFÉRENCES BIBLIOGRAPHIQUES}

Artz F.-B., 1938. - " L'éducation technique en France au XvIII" siècle (1700-1789)", Revue d'histoire moderne, tome XIII, n" 35 , oct.-déc.

Buisson F., 1888. - Dichionnaire pédagogique et instruction primaire, $1^{\text {rc }}$ partie, tome II, Paris, Hachette.

Calderon R., 1977. - "La leçon de choses ", Cahiers d'histoire de l'enseignement, n" 5.

Deforge Y., 1981. - Le graphisme technique, son histoire et son enseignement, Lyon, Champ Vallon.

Fouqué J., 1900. - La crise de l'apprentissage el les progrès de l'enseignement professionnel. Paris, Arthur Rousseau.

Gille B., 1978. - "L'histoire des techniques". Encyclopédie de la Pléiade. Paris, Gallimard.

Leblanc R., 1895. - L'enseignement professionnel au degré primaire. Paris, Larousse.

Léon A., 1961. - Histoire de l'éducation technique. Paris, Presses Universitaires de France.

- 1965. - Formation générale et apprentissage du métier. Paris, Presses Universitaires de France.

Malglaive G., Weber A., 1982. - "Théorie et pratique, approche critique de lalternance en pédagogie ", Revue française de pédagogie, n" 61, oct.-nov.-déc.

Mayeur F., 1981. - Histoire générale de l'enseignement et de l'éducation en France, tome III, Paris, Nouvelle Librairie de France.

Molinari J.-P., 1975. - Etude monographique et statistique de l'Ecole normale d'institutrices de Nantes, thèse de doctorat de $3^{\text {e }}$ cycle, Université de Nantes. $198 \mathrm{p}$.

Muel F., 1975. - "L'école obligatoire et l'invention de l'enfance anormale", Actes de la recherche en sciences sociales, 1, janv.

- 1977. - "Les instituteurs, les paysans et lordre républicain", Actes de la recherche en sciences sociales, nov.

Noiriel G., 1986. - Les ouvriers dans la société française (XIX'-XX' siècles), Paris, Le Seuil.

Offerlé M., 1984. - "lllégitimité et légitimation du personnel politique ouvrier en France avant $1914 »$, Annales, 39 (4), pp. 681-713.

Panthier A., 1906. - Enquête historique sur l'enseignement manuel dans les écoles techniques, Paris.

Paquier J.-B., 1908. - L'enseignement professionnel en France. Paris, A. Colin.

Prost A., 1968. - Histoire de l'enseignement en France (1800-1967), Paris. A. Colin.

Rougier-Pintiaux P., 1983. - « L'école d'apprentissage du Havre : une tentative d'éducation populaire". Revue française de sociologie, $24(4), \mathrm{pp} .653-678$.

Salomon O., s.d. - Le travail manuel à l'école primaire. Elude pédagogique (traduit du suédois sous la direction de G. Salicis), Paris.

Sandrin J., 1982. - Enfants trouvés, enfants ourriers (xvIr-XIX siecles), Paris, Aubier.

Testanière J., 1982. - Les enfants des milieux populaires et l'école. Une pédagogie populaire est-elle possible?, thèse de doctorat d'Etat, Université de Paris iv. $624 \mathrm{p}$.

Vincent G., 1972. - "Histoire et structure du système scolaire français : lenseignement primaire ", Revue française de sociologie, 13 (1), pp. 59-79. 